\title{
Spectral Integration in the Inferior Colliculus of the Mustached Bat
}

\author{
Scott A. Leroy and Jeffrey J. Wenstrup \\ Department of Neurobiology and Pharmacology, Northeastern Ohio Universities College of Medicine, Rootstown, Ohio \\ 44272-0095
}

\begin{abstract}
Acoustic behaviors including orientation and social communication depend on neural integration of information across the sound spectrum. In many species, spectral integration is performed by combination-sensitive neurons, responding best when distinct spectral elements in sounds are combined. These are generally considered a feature of information processing in the auditory forebrain. In the mustached bat's inferior colliculus (IC), they are common in frequency representations associated with sonar signals but have not been reported elsewhere in this bat's IC or the IC of other species. We examined the presence of combination-sensitive neurons in frequency representations of the mustached bat's IC not associated with biosonar. Seventyfive single-unit responses were recorded with the best frequencies in 10-23 or $32-47 \mathrm{kHz}$ bands. Twenty-six displayed single excitatory tuning curves in one band with no additional responsiveness to a second signal in another band. The remaining 49 re-
\end{abstract}

sponded to sounds in both $10-23$ and $32-47 \mathrm{kHz}$ bands, but response types varied. Sounds in the higher band were usually excitatory, whereas sounds in the lower band either facilitated or inhibited responses to the higher frequency signal. Interactions were usually strongest when the higher and lower frequency stimuli were presented simultaneously, but the strength of interactions varied. Over one-third of the neurons formed a distinct subset; they responded most sensitively to bandpass noise, and all were combination sensitive. We suggest that these combination-sensitive interactions are activated by elements of mustached bat social vocalizations. If so, neuronal integration characterizing analysis of social vocalizations in many species occurs in the IC.

Key words: auditory pathways; bat; combination sensitive; complex sounds; frequency integration; inferior colliculus; mustached bat; spectral integration
Acoustically guided behavior requires analyses of spectrally and temporally complex signals. The auditory system first decomposes sounds into their spectral components at the cochlea and then transmits the results of these analyses through frequency-tuned neurons of the auditory nerve. Further information processing of complex sounds uses the reverse of this spectral analysis, involving neuronal integration across spectral elements in sounds. For complex vocal signals, such neuronal integration is characterized by temporally sensitive facilitatory or inhibitory interactions between responses to distinct spectral elements. Sometimes called combination sensitive, these responses have been described in a variety of vertebrates from frogs to birds to mammals and are thought to contribute to selective responses to complex vocalizations (Suga et al., 1978, 1983; Fuzessery and Feng, 1983; Schuller et al., 1991b; Margoliash and Fortune, 1992; Olsen, 1992; Rauschecker et al., 1995; Ohlemiller et al., 1996; Doupe, 1997). It has been further suggested that combination-sensitive neurons underlie the encoding of phonemic elements of speech sounds (Suga, 1996; Sussman et al., 1998). Neural interactions that create these response properties are generally thought to originate in the auditory forebrain (Olsen, 1992; Winer et al., 1995; Rauschecker, 1998).

In the auditory cortex of the mustached bat (Pteronotus parnellii), combination-sensitive responses occur commonly among neurons tuned to frequency ranges of the bat's sonar vocalizations (see Fig. 1A). Most display facilitated responses to combinations of elements in the sonar call and returning echoes that may extract information about target features such as distance and movement

Received March 27, 2000; revised July 28, 2000; accepted Aug. 24, 2000.

This work was supported by the National Institute on Deafness and Other Communication Disorders, National Institutes of Health Grant 5 R01 DC 00937. We thank C. V. Portfors for helpful comments on this manuscript, C. D. Grose for technical assistance, F.-M. Chen for software, and the Natural Resources Conservation Authority of Jamaica for permission to collect bats.

Correspondence should be addressed to Dr. Jeffrey J. Wenstrup, Department of Neurobiology and Pharmacology, Northeastern OH Universities College of Medicine, 4209 State Route 44, Rootstown, OH 44272-0095. E-mail: jjw@neoucom.edu.

Dr. Leroy's present address: Abbott Laboratories, Building AP32-LL, 200 Abbott Park Road, Abbott Park, IL 60064.

Copyright (C) 2000 Society for Neuroscience $0270-6474 / 00 / 208533-09 \$ 15.00 / 0$
(O’Neill and Suga, 1979; Suga et al., 1983). Recently, it has become clear that such neurons are abundant in the mustached bat's inferior colliculus (IC) (Mittmann and Wenstrup, 1995; Yan and Suga, 1996; Portfors and Wenstrup, 1999a). Their presence in the auditory midbrain may be understandable in the context of sonar behavior, in which flight adjustments in response to target movement must be made within the 1-2 sec duration of an interception sequence. Projections of the IC to premotor centers could provide highly processed information useful for rapid changes in vocalization or flight (Schweizer, 1981; Frisina et al., 1989; Schuller et al., 1991a; Wenstrup et al., 1994; Casseday and Covey, 1996).

Is the presence of these sonar-related neurons in the IC an exception, or does similar spectral integration occur among neurons analyzing other types of complex acoustic signals? This report examines whether combination-sensitive responses occur in frequency representations of the mustached bat's IC outside those used in biosonar (see Fig. 1A). We found that the majority of neurons responding to these frequency bands (10-23 and 32-47 $\mathrm{kHz}$ ) displayed combination-sensitive responses. This indicates that the integration of information from distinct spectral elements in sounds is performed by a wide range of IC neurons and is not an exclusive property of neurons analyzing biosonar information. We suggest that many of these response properties are well suited for analyses of the mustached bat's social vocalizations (Kanwal et al., 1994). If so, neuronal integration characterizing analysis of social vocalizations in many species occurs in the auditory midbrain, not just in the auditory cortex as is commonly supposed.

\section{MATERIALS AND METHODS}

Single-unit recordings were obtained from the IC in awake greater mustached bats (P. parnellii parnellii). Thirteen bats, captured in Jamaica, West Indies, provided the data used in this report. All procedures on the bats followed protocols approved by the Institutional Animal Care and Use Committee of the Northeastern Ohio Universities College of Medicine.

Surgical preparation. At least $1 \mathrm{~d}$ before surgery, bats were placed in a holding cage with tetracycline in their drinking water. For surgery, the bats were anesthetized with methoxyflurane (Metofane; Pitman-Moore, Inc., Mundelein, IL) in combination with sodium pentobarbital (5 mg/kg, i.p.; Nembutal; Abbott Labs, Irving, TX) and acepromazine ( $2 \mathrm{mg} / \mathrm{kg}$, i.p.; Med-Tech, Inc., Buffalo, NY). A midline incision was made over the dorsal surface of the skull, and the skin and muscles were retracted laterally. A 
tungsten ground electrode was then cemented into the skull over the cerebral cortex on the side opposite to the exposed IC. A metal pin was glued to the skull to hold the head in the stereotaxic apparatus and ensure the proper position of the brain during experiments. After a topical antibiotic and local anesthetic (lidocaine; Elkins-Sinns, Cherry Hill, NJ) were applied to the wound, the bat was returned to the holding cage and allowed to recover for at least $1 \mathrm{~d}$ before physiological recording.

Acoustic stimulation and physiological recording. On the first day of recording, bats were anesthetized with methoxyflurane, and a small piece of the skull $(<0.5 \mathrm{~mm}$ in diameter) was removed to expose the IC. During recording sessions, the animals were placed in a stereotaxic apparatus to orient the head in a standard position. The apparatus was housed in a heated and humidified sound-attenuating chamber, lined with polyurethane foam to reduce echoes. In addition, the surface of the stereotaxic apparatus was covered with cotton to reduce echoes. If a bat struggled or showed other signs of discomfort, it was returned to its holding cage. Between electrode penetrations, the bats were given water, and the exposed brain tissue was covered with petroleum jelly to keep it moist. Recording sessions generally lasted 4-6 hr.

Stimulus generation and data acquisition were computer-controlled. Two different tone or noise bursts (duration varied; $0.5 \mathrm{msec}$ rise-fall time; $3-4 / \mathrm{sec}$ ) were separately generated (WaveTek model 395), switched (Tucker-Davis Technologies model SW2), and attenuated (Tucker-Davis Technologies model PA4). The signals were combined (Tucker-Davis Technologies model SM3) and then sent to a power amplifier (Parasound model HCA-800II) and a speaker (Technics leaf tweeter) placed $10 \mathrm{~cm}$ away from the bat and $25^{\circ}$ into the sound field contralateral to the recording electrode.

The acoustic system was calibrated several times over the course of the experiments. A calibrated microphone (Brüel and Kjaer model 4135) was placed in the position of the bat's head during experiments. There was a smooth, consistent decrease of $2.7 \mathrm{~dB}$ per $10 \mathrm{kHz}$ from 10 to $120 \mathrm{kHz}$. The microphone output was digitized (National Instruments model NBA2000), and a fast Fourier transform was computed. Distortion components were not detectable $60 \mathrm{~dB}$ below the peak signal level.

Single-unit activity was recorded with micropipette electrodes filled with $3 \mathrm{M} \mathrm{KCl}$ and having resistances of $8-15 \mathrm{M} \Omega$. In many experiments, multiunit responses were also recorded to compare the single-unit responses with the surrounding population. Electrodes were positioned for recording in the IC by the use of surface landmarks and advanced with a hydraulic micropositioner (David Kopf Instruments model 650). Extracellular action potentials were amplified and then sent through a bandpass filter $(500-6000 \mathrm{~Hz})$ and a window discriminator (Frederick Haer and Company model 74-60-3). The output of the discriminator was then digitized at $10 \mathrm{kHz}$ for analysis of spike times (National Instruments model NB-MIO-16X). The laboratory software generated histograms, raster displays, and statistics on neural responses within a $100 \mathrm{msec}$ peristimulus window for 32 stimulus presentations. Usually, spike counts were based on the entire $100 \mathrm{msec}$ window. The duration of the window was reduced to 50 or $60 \mathrm{msec}$ if there was high spontaneous activity that obscured differences in response magnitude or for display purposes (e.g., see Figs. 3, 7). In no case did shortening the window duration eliminate an observable response. The output of the window discriminator was also sent to an oscilloscope and a speaker for audiovisual display and estimates of response thresholds (see below).

Neurons were stimulated with tone bursts or bandpass noise bursts (roll-offs exceeded $100 \mathrm{~dB}$ per octave). Signal duration was typically 30 msec but was changed for neurons that responded better to shorter (as short as $3 \mathrm{msec}$ ) or longer (up to $70 \mathrm{msec}$ ) stimuli. When a single unit was isolated, we obtained its best frequency, threshold, and tuning curve by monitoring the oscilloscope and audio monitor, i.e., audiovisually. We defined best frequency as the frequency requiring the lowest intensity to elicit stimulus-locked spikes and threshold as the lowest intensity required to elicit a consistent spike response. For units that were excited by sounds within two different frequency bands, we tuned the response in each frequency band and refer to a best high-frequency response and a best low-frequency response. For some neurons that responded poorly to tones, frequency tuning was tested with $5-\mathrm{kHz}$-wide noise bands.

By the use of a two-stimulus paradigm, single units were tested for sensitivity to combinations of tone or noise bursts as described previously (Portfors and Wenstrup, 1999a; Wenstrup, 1999). Sensitivity to delay between the low- and high-frequency signals was initially assessed audiovisually by varying the delay, usually in steps of $2 \mathrm{msec}$. If we noted a detectable change in response as a function of delay, quantitative data were collected at the delay that evoked the largest change in response. This was compared with the unit's response magnitude to the two stimuli presented separately. Quantitative data were then obtained as a function of delay. The range of delays tested included those in which the lowfrequency signal was presented first and the high-frequency signal was delayed and also those in which the high-frequency signal was presented first and the low-frequency signal was delayed. The delay between the lowand high-frequency signals that elicited the greatest response (or the least response in the case of an inhibited combination-sensitive effect) was defined as the neuron's best delay.

We then tuned the facilitated or inhibited response. For facilitated neurons, we tuned both the low- and high-frequency sounds. To assess the low frequencies that elicited a facilitated response, the high-frequency signal was held constant, and the low frequencies eliciting a facilitated response (a detectable change in the response rate evaluated audiovisually) at various intensities were recorded. Then the low-frequency signal was held constant, and the responses to the high frequencies were tuned across intensities. For inhibited neurons, we determined the range of low frequencies that inhibited the high-frequency response while the highfrequency sound was held constant. For both facilitated and inhibited responses, the sound held constant was typically presented at $10 \mathrm{~dB}$ above threshold.

Single-unit responses were considered to be combination sensitive if they met three criteria: (1) the response to the two signals presented together was $20 \%$ more (for facilitation) or $20 \%$ less (for inhibition) than the sum of responses to the signals presented separately, (2) the facilitatory or inhibitory interactions were tuned to clearly distinct frequency bands (separated by a half octave or more), and (3) the facilitatory or inhibitory interaction displayed temporal sensitivity as revealed in delay tests. The degree of facilitation or inhibition was quantified as the index of interaction $(I)$, according to the following formula: $I=(\mathrm{Rc}-\mathrm{Rl}-\mathrm{Rh}) /(\mathrm{Rc}+$ $\mathrm{Rl}+\mathrm{Rh}$ ), where $\mathrm{Rc}, \mathrm{Rl}$, and $\mathrm{Rh}$ are the neuron's responses to the combination of the low- and high-frequency signals, the low-frequency signal alone, and the high-frequency signal alone, respectively. An interaction index value of 0.09 corresponds to $20 \%$ facilitation, the criterion for combination-sensitive facilitation. Negative numbers indicate combination-sensitive inhibition. Interaction index values of 1 and -1 indicate maximum facilitation and inhibition, respectively. These criteria are identical to those used in similar studies of sonar-related combinationsensitive neurons in the IC (Portfors and Wenstrup, 1999a) and medial geniculate body (MGB) (Wenstrup, 1999).

To test for combination sensitivity unrelated to the processing of sonar signals, we recorded from the $10-23$ and $32-47 \mathrm{kHz}$ representations of the IC. Among Jamaican mustached bats, the frequency range of the sonar fundamental emitted by resting bats is $\sim 24-31 \mathrm{kHz}$ (see Fig. $1 A$ ), differing slightly among individuals (Kobler et al., 1985; Suga et al., 1987). In flight, sonar echoes are shifted upward in frequency as a function of the bat's flight speed toward the echo source. Mustached bats compensate for upward Doppler frequency shifts by lowering the frequency of emitted signals (Schnitzler, 1970; Keating et al., 1994). In the laboratory, flight velocities are $<5 \mathrm{~m} / \mathrm{sec}$ (Schnitzler, 1970; Lancaster et al., 1992). Although these bats may fly faster in natural habitats, speeds $>10 \mathrm{~m} / \mathrm{sec}$ are unlikely (Norberg, 1987). Even if mustached bats compensated completely for frequency shifts introduced by a flight velocity of $10 \mathrm{~m} / \mathrm{sec}$, the lower frequency of the emitted signal would extend down only to $22 \mathrm{kHz}$ for the fundamental and $44 \mathrm{kHz}$ for the second harmonic. This suggests that neural responses tuned $<22 \mathrm{kHz}$ and between 33 and $44 \mathrm{kHz}$ are not involved in the analysis of sonar signals.

Histological procedures. In some electrode penetrations, a tracer was deposited to mark recording sites. Electrodes were filled with dextran conjugates (dextran-rhodamine or biotin dextran amine; Molecular Probes, Eugene, OR) in $0.9 \% \mathrm{NaCl}$, iontophoresed with pulsed current $(+5 \mu \mathrm{A} ; 7 \mathrm{sec}$ on and $7 \mathrm{sec}$ off $)$ for at least $6 \mathrm{~min}$, or $1 \%$ Fluoro-Gold (Fluorochrome, Inc., Englewood, $\mathrm{CO}$ ) in $0.9 \% \mathrm{NaCl}$, iontophoresed with pulsed current $(+1 \mu \mathrm{A} ; 7 \mathrm{sec}$ on and $7 \mathrm{sec}$ off $)$ for $5 \mathrm{~min}$. In all but one animal, each marked penetration used a different tracer.

The animals were perfused within $12 \mathrm{~d}$ of deposits. After the bat was deeply anesthetized with Nembutal $(75 \mathrm{mg} / \mathrm{kg}$, i.p.) and reflexes were eliminated, the chest was opened, and the bat was perfused through the heart with phosphate buffer and $10 \%$ Formalin. The head was removed, blocked, and refrigerated overnight in $30 \%$ sucrose. The brain was sectioned with a freezing microtome, usually at $40 \mu \mathrm{m}$. The sections were collected in chilled $0.1 \mathrm{M}$ phosphate buffer and rinsed in $0.05 \mathrm{M}$ phosphate buffer before mounting on slides. At least one of the three series was stained with cresyl violet to reveal cytoarchitecture.

The majority of single units described in this report responded to sounds in both the $10-23$ and $32-47 \mathrm{kHz}$ bands. Because most units were not histologically localized, we used physiological and depth criteria to determine whether a unit was located within the $10-23$ or $32-47 \mathrm{kHz}$ frequency band representations of the tonotopically organized IC. This analysis is based on previous studies in mustached bats showing that frequencies in the $\sim 10-59 \mathrm{kHz}$ range are represented in the anterolateral division of the IC (Zook et al., 1985; O'Neill et al., 1989). Here, anatomically defined fibrodendritic laminae (Zook et al., 1985) and physiologically defined frequency-band laminae (O'Neill et al., 1989) extend dorsoventrally and mediolaterally, their edges curving rostrally. There is an orderly progression from the highest frequencies located more caudally $(59 \mathrm{kHz})$ to lower frequencies represented more rostrally. Our penetrations, which were angled $5-25^{\circ}$ dorsocaudal to ventrorostral, are expected to encounter decreasing best frequencies with increasing depth, as have other studies using similar electrode orientations (Wenstrup et al., 1994; Wenstrup and Grose, 1995). All single units in the present study were recorded at depths of $125-1975 \mu \mathrm{m}$, consistent with IC recordings of $10-50 \mathrm{kHz}$ responses in these previous studies using similar electrode angles.

To localize a single-unit response within the physiological tonotopic organization, we required the penetration to show a descending progression of best frequencies combined with the presence of singly tuned responses (single unit or multiunit) to $24-31 \mathrm{kHz}$, the frequency representation that intervenes between the $10-23$ and $32-47 \mathrm{kHz}$ bands. Thus, if a combinatorial unit responding to both the $10-23$ and $32-47 \mathrm{kHz}$ bands 

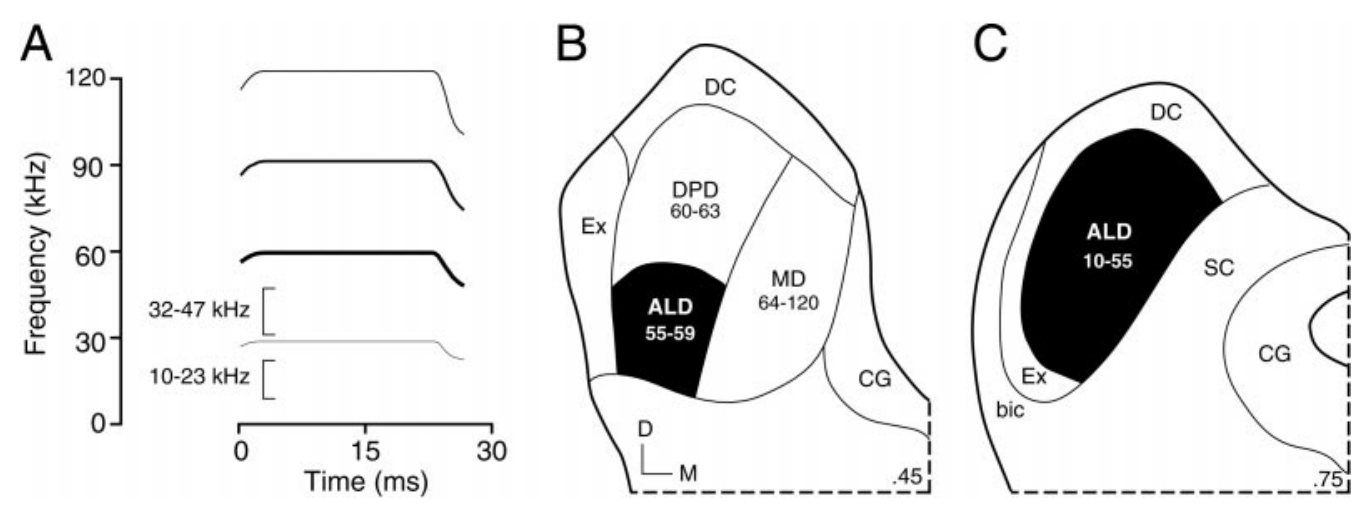

Figure 1. A, Schematic sound spectrogram of the mustached bat's biosonar call. The frequency bands labeled by the brackets (10-23 and $32-47 \mathrm{kHz}$ ) are analyzed by neurons in the present report. $B, C$, Schematic illustration of the organization of the mustached bat's central nucleus of the inferior colliculus (ICC) in transverse sections. Numbers at the bottom right of the sections indicate fractions of the caudal-to-rostral dimension of the IC; the section in $B$ is more caudal than the section in $C$. Numbers below abbreviations indicate the frequency representation (in kiloHertz) of each ICC subdivision. In the present study, single units were recorded from the anterolateral division $(A L D)$ of the ICC (blackened region). In $A L D$, tonotopic representation advances from $\sim 10 \mathrm{kHz}$ at the rostral tip to frequencies up to $59 \mathrm{kHz}$ more caudally (Zook et al., 1985; O'Neill et al., 1989). bic, Brachium of the inferior colliculus; $C G$, central gray; $D$, dorsal; $D C$, dorsal cortex of the inferior colliculus; $D P D$, dorsoposterior division or the ICC; $E x$, external nucleus of the inferior colliculus; $M$, medial; $M D$, medial division of the ICC; $S C$, superior colliculus.

occurred more superficially in the penetration than did a $24-31 \mathrm{kHz}$ response, we concluded that the combinatorial unit was in the $32-47 \mathrm{kHz}$ representation. If it had occurred deeper, we would conclude that the combinatorial unit was in the $10-23 \mathrm{kHz}$ representation. A similar analysis was performed for neurons responding to only one of the $10-23$ or 32-47 $\mathrm{kHz}$ frequency bands. By the use of these criteria, 38 of 75 single-unit responses were localized within the physiological tonotopic organization of the IC.

\section{RESULTS}

The anterolateral division of the mustached bat's IC represents frequencies in the $10-59 \mathrm{kHz}$ range (Fig. $1 B, C$ ). This range includes frequency bands used in biosonar $(24-31 \mathrm{kHz}$, first sonar harmonic; 48-59 $\mathrm{kHz}$, second harmonic of the frequencymodulated sonar component) as well as frequency bands below and above the first sonar harmonic (10-23 and $32-47 \mathrm{kHz}$, respectively) (Fig. 1A). This report describes single-unit recordings of 75 neurons tuned to signals in either the $10-23$ or $32-47 \mathrm{kHz}$ frequency bands; we examined tuning to multiple frequency bands and sensitivity to combinations of tonal or noise stimuli. Multiunit responses and single units tuned to other frequency bands were also examined to establish the location of the single-unit responses described here within the tonotopic map of the IC.

Of the 75 single units, $26(35 \%)$ displayed a single excitatory tuning curve with their best frequencies in either the $10-23 \mathrm{kHz}$ $(n=6)$ or $32-47 \mathrm{kHz}(n=20)$ frequency bands (Fig. 2). Furthermore, in the presence of a sound at their best excitatory frequency, these neurons showed no additional tuned responsiveness, either facilitatory or inhibitory, to sounds over a wide range of frequencies, even when tested over a range of delays between the two sounds. On the basis of our definition (see Materials and Methods), these neurons were not combination sensitive. In contrast, the majority of neurons in our sample (49 of $75,65 \%$ ) had excitatory responses tuned to one or both of the $10-23$ or $32-47 \mathrm{kHz}$ bands but in addition showed facilitatory or inhibitory interactions when stimuli in the two frequency bands were presented together (Fig. 2). Single units with facilitatory interactions (57\%) were slightly more common than were those with inhibitory interactions $(43 \%)$.

\section{Facilitatory and inhibitory combination-sensitive interactions}

Responses of a single unit in Figure $3 A-C$ illustrate fundamental properties of combination-sensitive facilitation. In response to single tone burst stimuli, this unit displayed a sensitive excitatory tuning curve, centered at $40.4 \mathrm{kHz}$ with the threshold at $19 \mathrm{~dB}$ sound pressure level (SPL). Its responses to signals in the 10-23 $\mathrm{kHz}$ band were weak and insensitive, with threshold responses near $20 \mathrm{kHz}$ obtained only at levels exceeding $80 \mathrm{~dB}$ SPL (Fig. 3A, unfilled circles and dashed lines). However, the combination of high-

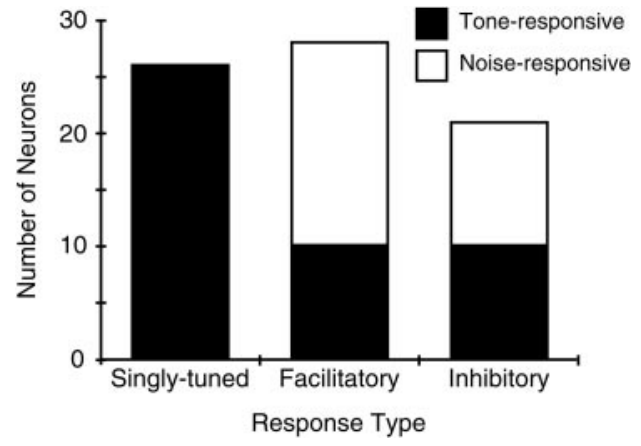

Figure 2. Categories of response to combinations of tone or noise bursts among single units tuned to $10-23$ and $32-47 \mathrm{kHz}$ frequency bands. Singly tuned neurons had an excitatory response tuned to only one frequency band. Facilitatory neurons responded to the combination of two signals from the $10-23$ and $32-47 \mathrm{kHz}$ bands at a level higher than the sum of responses to the separate signals (see Materials and Methods). Inhibitory neurons showed an excitatory response to one of these frequency bands that was inhibited by signals in the other band. Tone-responsive and Noise-responsive refer to single units that displayed better responses (lower threshold or more spikes) to tone bursts or bandpass noise bursts, respectively.

and low-frequency stimuli revealed a low-threshold, facilitating input that was sharply tuned to $19.2 \mathrm{kHz}$ (Fig. $3 A$, filled circles and solid lines). The drop in the threshold response to the lower frequency signal, when presented together with the facilitating higher frequency signal, was substantial, $61 \mathrm{~dB}$ for the neuron in Figure $3 A$. The facilitated response to the high-frequency signal, also sharply tuned, decreased in threshold but only by $10 \mathrm{~dB}$. The unit did not respond to signals in the $24-31 \mathrm{kHz}$ band, the frequency of the first sonar harmonic, in either single- or two-tone presentations. In addition to its effect on response threshold, the facilitation also increased response magnitude. The response to combined tone bursts was $123 \%$ greater than the sum of responses to the higher and lower frequency signals presented separately (Fig. 3B), corresponding to a facilitation index value of 0.38 . This facilitation was also dependent on the relative timing of the two signals. Strong facilitation was only obtained when the higher frequency signal was presented simultaneously with or $2 \mathrm{msec}$ after the lower frequency signal (Fig. $3 C$ ). The frequency- and time-dependent facilitation shown for the neuron in Figure $3 A-C$ satisfies the criteria for combination sensitivity described in Materials and Methods.

For 21 units, the effect of signals in one of the $10-23$ or $32-47$ $\mathrm{kHz}$ frequency bands was to inhibit responses to signals in the other band (Fig. 2). The unit in Figure $3 D-F$ had a sensitive excitatory tuning curve in the higher frequency band, tuned to $34.9 \mathrm{kHz}$ (Fig. 
Figure 3. Combinatorial interactions of single units responsive to $10-23$ and $32-47 \mathrm{kHz}$ frequency bands. $A-C$, Responses of a facilitated combination-sensitive unit. $A$, Frequency tuning in response to single tones and combinations of tones. Dashed lines and unfilled circles indicate responses to singletone bursts. Solid lines and filled circles indicate tuning curves obtained in the presence of a second tone burst, the frequency and intensity of which are indicated by the $X$ placed within the other tuning curve. Each $X$ also indicates the frequencies and intensities of signals used in the tests shown in $B$ and $C$. The unit did not respond to frequencies in the fundamental sonar harmonic (24-31 $\mathrm{kHz}$ ) at any sound level tested. $B$, Peristimulus time histograms (PSTHs; 32 stimulus presentations) showing a facilitated response when signals were presented simultaneously. Signal timing and duration $(3 \mathrm{msec})$ are indicated by the unfilled bars (higher frequency signals) and solid bars (lower frequency signals). $C$, Temporal sensitivity of facilitation. Facilitation only occurred when the two signals were presented near the $0 \mathrm{msec}$ delay. Error bars indicate $95 \%$ confidence limits. Separate data points indicate responses of single tones at the indicated frequencies. $D-F$, Responses of an inhibited combinationsensitive unit. $D$, Frequency tuning in response to single tones and combinations of tones. The blackened curve indicates tuning of an inhibitory response that suppresses the excitatory response to the higher frequency signal marked by the $X$. The $X$ symbols also mark the frequencies and intensities of test signals used in the tests shown in $E$ and $F$. $E$, PSTHs demonstrating moderate suppression of the higher frequency response by the lower frequency signal presented $4 \mathrm{msec}$ before the higher frequency signal. The signal duration was 30 msec. $F$, Temporal sensitivity of inhibition.
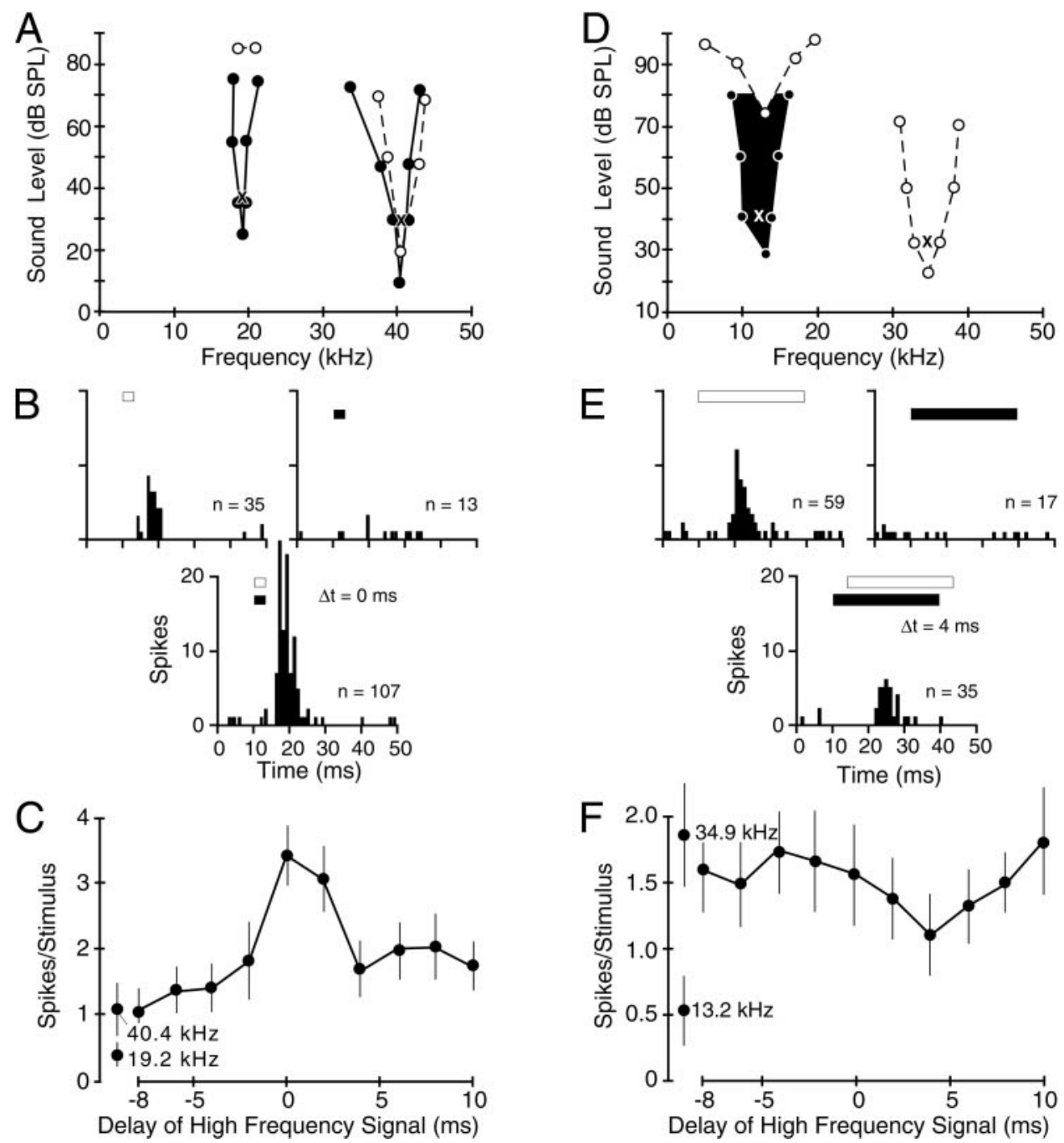

$3 D)$. Excitatory responses to the $10-23 \mathrm{kHz}$ band were tuned near $13 \mathrm{kHz}$ but only obtained at intensities $>70 \mathrm{~dB}$ SPL. The combination of low-intensity signals in both frequency bands revealed a low-frequency inhibitory effect, sharply tuned at $13.2 \mathrm{kHz}$ with a low threshold, which reduced the excitatory response to the highfrequency stimulus. At an intensity $9 \mathrm{~dB}$ above the threshold for inhibition, the lower frequency signal suppressed the response to the higher frequency signal by $41 \%$, with an index of inhibition of -0.37 (Fig. $3 E$ ). As with facilitatory interactions, the inhibitory interactions were sensitive to the timing of the two signals. In the unit in Figure $3 D-F$, the inhibitory effect was maximal when the high-frequency signal followed the lower frequency signal by 4 msec (Fig. $3 F$ ). However, most inhibitory units showed the greatest suppression when the inhibitory stimulus was presented simultaneously with the excitatory stimulus (see Temporal sensitivity).

A substantial number of single units (29 of 75, 39\%) responded well to bandpass noise $(5-20 \mathrm{kHz})$. For these neurons, responses to tonal stimuli either alone or in combination were poor. Among 15 neurons for which tone and noise thresholds were compared, thresholds to tones were on average $>38 \mathrm{~dB}$ higher than thresholds for noise stimuli, on the basis of decibel attenuation values. The average difference in the threshold was probably higher, because no tone threshold could be obtained in 11 of the 15 tested neurons. Responses to noise stimuli and combinations of noise stimuli could nonetheless be tuned in frequency. Figure 4 shows the tuning properties of two such neurons, obtained by presenting $5-\mathrm{kHz}$-wide noise bands at different center frequencies. As with combinationsensitive neurons that responded well to tones, these neurons showed either facilitatory (Fig. 4A) or inhibitory (Fig. 4B) effects of the lower frequency stimulus. For the facilitated neuron in Figure $4 A$, the facilitatory interaction reduced the threshold to the lowfrequency stimulus by $46 \mathrm{~dB}$, whereas the decrease in threshold for the high-frequency response was smaller $(8 \mathrm{~dB})$. A striking feature of noise-responsive neurons is that all displayed combinationsensitive interactions between signals in the $10-23$ and $32-47 \mathrm{kHz}$ bands; 18 units showed facilitatory interactions, and 11 units showed inhibitory interactions (Fig. 2).

\section{Frequency sensitivity}

Tuning curves in Figures 3 and 4 show that these neurons displayed two distinct frequency sensitivities that did not overlap, except possibly at high sound levels. Figure $5 A$ shows the best low and high frequencies for neurons responding to signals in both frequency bands. Low best frequencies ranged from 12 to $23 \mathrm{kHz}$, whereas high best frequencies ranged from 32 to $47 \mathrm{kHz}$. Only one neuron was tuned to exact harmonics, although several were tuned very close to a harmonic relationship. For the majority, the best highfrequency response was tuned more than an octave above the best low-frequency response. One implication of these results is that few of these combination-sensitive interactions would be activated by signals within the frequency bands of biosonar signals.

In most neurons, the response to sounds in the higher frequency band was greater than that to sounds in the lower frequency band. At the sound levels tested, in which the lower frequency signal was usually more intense, 21 of 28 facilitated neurons responded more strongly to the higher frequency signal, 4 neurons responded to the lower and higher frequencies at the same magnitude, and only 3 facilitated neurons responded more strongly to the lower frequency 

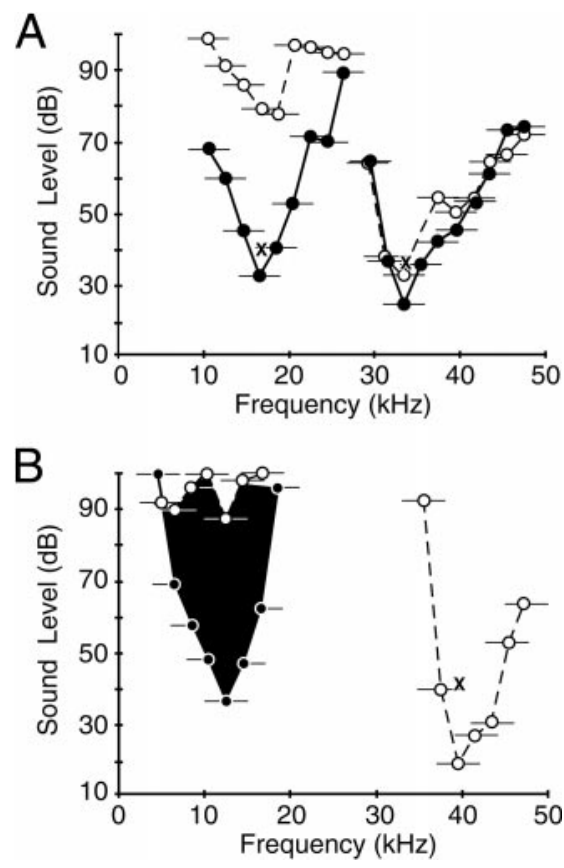

Figure 4. Tuning curves for combination-sensitive single units that respond best to noise stimuli. Thresholds were obtained with $5-\mathrm{kHz}$-wide noise bands, indicated in the figure by the circles (center frequency) and horizontal lines (bandwidth). Dashed lines and unfilled circles indicate responses to single noise bursts. Solid lines and filled circles indicate tuning curves obtained in the presence of a second noise burst, the center frequency and intensity of which are indicated by the $X$ placed within the other tuning curve. The blackened curve shows tuning of an inhibitory response as described in Figure 3. A, Single unit facilitated by signals in the $10-23$ and $32-47 \mathrm{kHz}$ frequency bands. $B$. Single unit excited by signals in the higher frequency band but inhibited by signals in the lower frequency band. For both units, combination effects were documented when signals were presented simultaneously ( $0 \mathrm{msec}$ delay). The units did not respond to tonal stimuli in the $10-50 \mathrm{kHz}$ range at levels as high as $80 \mathrm{~dB}$ SPL, the highest tested.

signal. For inhibited neurons, 18 of 21 neurons were excited by the higher frequency signal and suppressed by the lower frequency signal. The greater excitatory responses to the higher frequency signal may be related to the probable location of these neurons in the ICC representation of the higher frequency band (see below).

\section{Strength of interactions}

The strength of combination-sensitive interactions, as expressed by facilitation or inhibition indexes, varied among the population (Fig. $5 B)$. For 28 facilitatory neurons, the average facilitation index was 0.32 (SD, 0.24), corresponding to a facilitated response that was $95 \%$ greater than the sum of responses to the low- and highfrequency stimuli presented separately. Strengths of facilitation ranged from 20 to $1200 \%$ (facilitation index values of $0.09-0.98$ ). For 21 inhibitory neurons, the average inhibitory index was -0.36 (SD, 0.21), corresponding to suppression of the excitatory response by $53 \%$. Strengths of inhibition ranged from 20 to $97 \%$ (inhibitory index values of -0.11 to -0.94 ). However, inhibitory index values do not indicate the maximum effect of inhibition on these neurons, because the inhibiting stimulus was typically presented at $10 \mathrm{~dB}$ above the threshold for inhibition. Comparing the noise- and tone-responsive units, there was no significant difference in the mean facilitatory index (unpaired $t$ test; $\mathrm{df}=26 ; p>0.5$ ) or the mean inhibitory index (unpaired $t$ test; $\mathrm{df}=19 ; p>0.5$ ). However, all noise-responsive neurons showed combination sensitivity, whereas many tone-responsive units were singly tuned (Fig. 2).

\section{Temporal sensitivity}

The facilitatory interactions in responses to the two frequency bands showed clear selectivity for the timing of the two signals (Figs. 3C, 6). Most facilitatory responses were best when the two
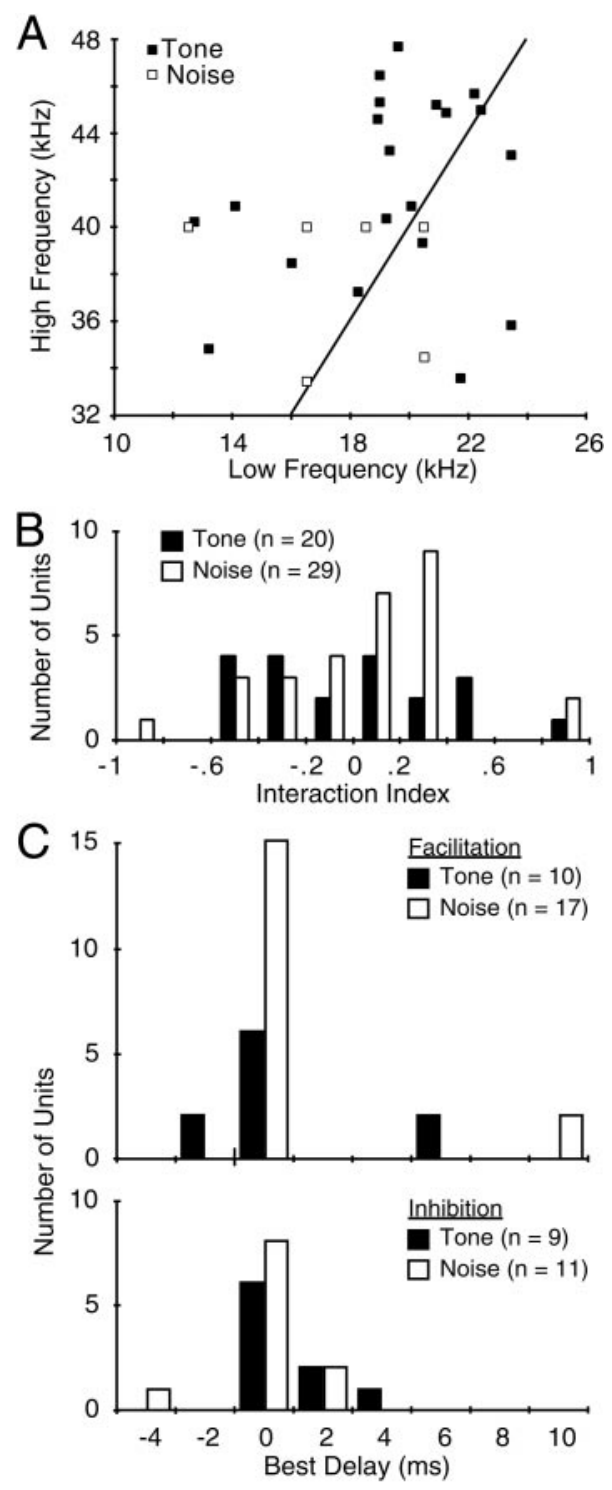

Figure 5. Aspects of combinatorial interactions among the recorded population of single units. $A$, Frequency tuning of combination-sensitive interactions. Tone indicates tone-responsive units, whereas Noise indicates units responding better to bandpass noise. The line plots an exact fundamentalsecond harmonic relationship. Most noise-sensitive units are not included because their best frequencies were not measured. These were stimulated with noise bursts including most of the $10-23$ or $32-47 \mathrm{kHz}$ bands. $B$, Strength of interaction, as measured by the interaction index. Only units with index values of 0.09 and greater (facilitation) or -0.11 or less (inhibition) are shown. $C$, Best delays among single units showing combinationsensitive facilitation (top) or inhibition (bottom).

signals were presented simultaneously, e.g., 0 msec delay (Fig. 5C). However, the sharpness of this temporal selectivity, or delay tuning, was variable. Figure 6 offers a particularly sharp contrast. The unit in Figure $6 A$ responded well only when the two $50 \mathrm{msec}$, narrowband noise bursts were presented within $2 \mathrm{msec}$ of each other. In contrast, the unit in Figure $6 B$, likewise stimulated with relatively long-duration $(30 \mathrm{msec})$ signals, responded well as long as some overlap occurred between the high- and low-frequency signals. Such results suggest a range of temporal integration properties across the population of facilitated units.

Inhibitory combination-sensitive interactions were also sensitive to timing (Figs. $3 F, 7$ ). Most inhibitory units showed the greatest suppression when the inhibitory stimulus was presented simultaneously with the excitatory stimulus (Fig. 5C). As with facilitated units, there was variability in the width of the inhibitory delay function. The unit in Figure 7 showed very sharp temporal sensitivity of inhibition, whereas that in Figure $3 F$ was broader. 

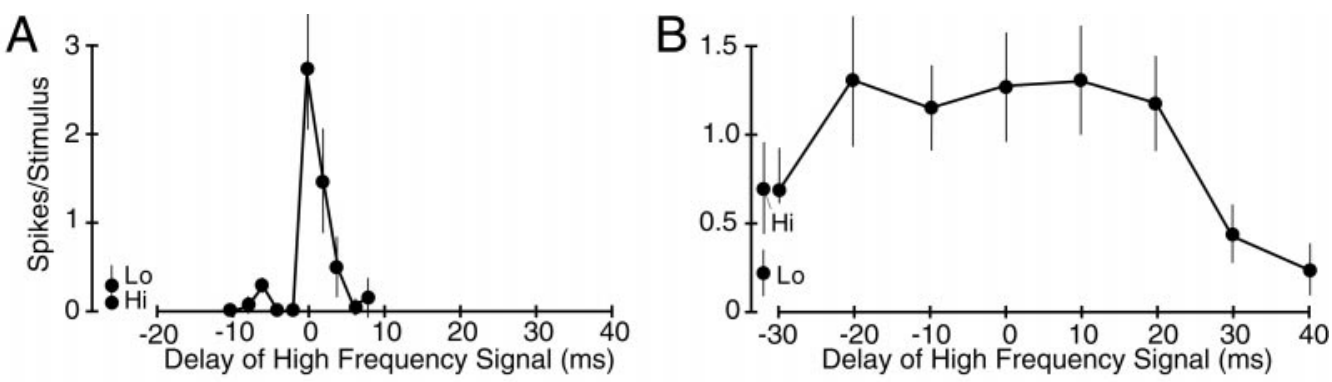

Figure 6. Differences in temporal sensitivity among facilitated combinationsensitive single units. $A$, Strongly facilitated neuron with best delay at $0 \mathrm{msec}$. Note the narrow temporal selectivity despite the use of long-duration signals $(50 \mathrm{msec})$. This noise-responsive unit was stimulated with $10-23$ and $35-45$ $\mathrm{kHz}$ noise bands. $B$, Facilitated unit with very broad delay sensitivity. This noise-responsive unit was stimulated with $12-22$ and $35-45 \mathrm{kHz}$ noise bands. See Figure 3 for an explanation of figure conventions and symbols.
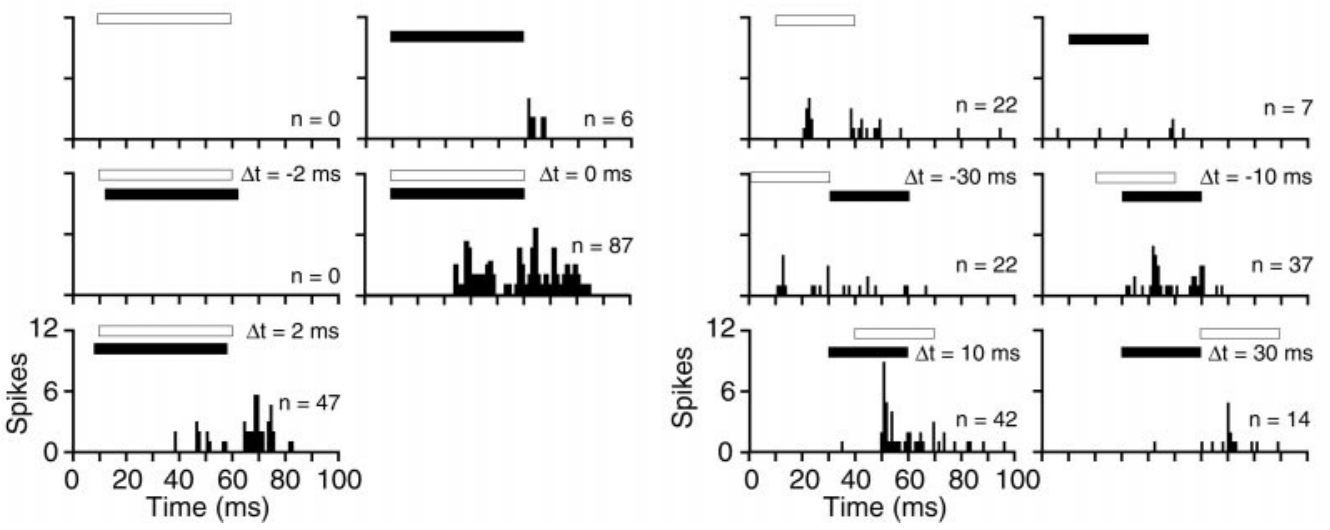
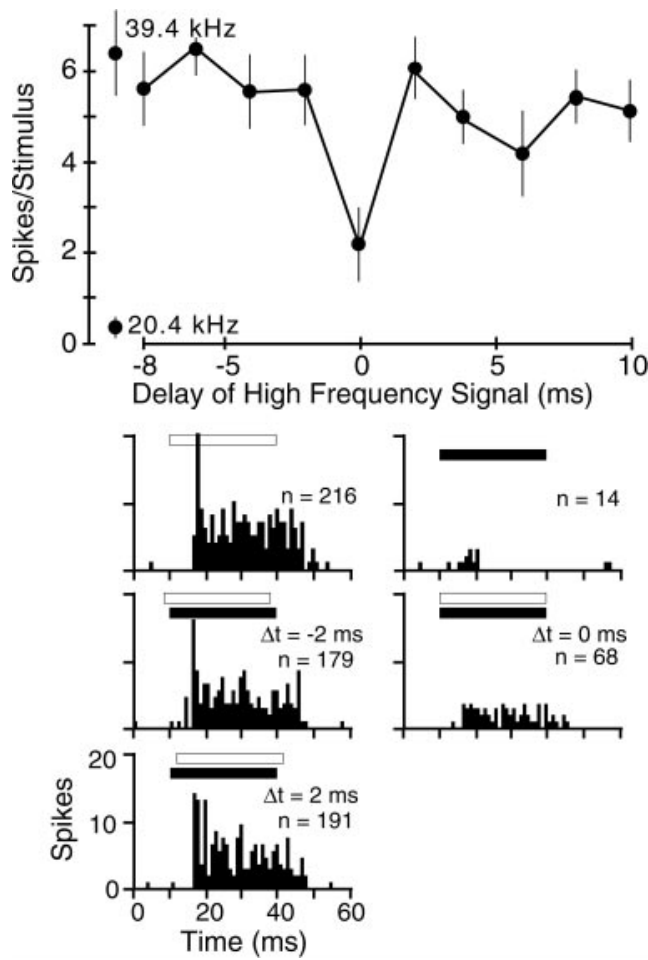

Figure 7. Sharp temporal sensitivity of low-frequency inhibition. In contrast to the inhibitory unit in Figure $3 F$, this single unit displayed strong inhibition with sharp temporal selectivity. Inhibition was eliminated when the relative timing of tone bursts was changed by $2 \mathrm{msec}$. See Figure 3 for an explanation of figure conventions and symbols.

\section{Topographic features}

Tracer deposits placed in some penetrations showed that both tone- and noise-responsive combination-sensitive neurons were located in the ICC (Fig. 8). For 38 of the 75 single units in our sample, there were sufficient data from other responses in the same penetration to determine the unit's location within the tonotopic organization of the IC (see Materials and Methods). To make this determination, we required the penetration to show a descending progression of best frequencies in the dorsocaudal to ventrorostral penetration, combined with the presence of singly tuned responses (single unit or multiunit) to $24-31 \mathrm{kHz}$, the frequency representation that intervenes between the $10-23$ and $32-47 \mathrm{kHz}$ bands (Fig. 8). For singly tuned neurons, 17 of 18 localized single units were in the tonotopic representation of $32-47 \mathrm{kHz}$ (e.g., Fig. $8 A$ ). For combination-sensitive responses, all 20 localized units were in the $32-47 \mathrm{kHz}$ representation (e.g., Fig. 8). These results show that combination-sensitive responses are common in the $32-47 \mathrm{kHz}$ representation. Because so few neurons were localized to the 10-23 $\mathrm{kHz}$ representation, it is unclear whether it too contains combination-sensitive response properties. All combinationsensitive neurons localized to the $32-47 \mathrm{kHz}$ tonotopic representation in the IC had higher magnitudes of response to signals in that frequency band than to signals in the $10-23 \mathrm{kHz}$ frequency band, at the sound intensities used to assess combinatorial properties.

\section{DISCUSSION}

This study describes integrative neurons in the IC of mustached bats responding to combinations of acoustic signals in two frequency bands, $10-23$ and $32-47 \mathrm{kHz}$. Previous studies showed that combination-sensitive neurons are common in IC frequency representations analyzing biosonar vocalizations (Mittmann and Wenstrup, 1995; Yan and Suga, 1996; Portfors and Wenstrup, 1999a). The present results show that the type of frequency-time integration performed by combination-sensitive neurons is widespread throughout the mustached bat's IC and is not a feature unique to the analysis of biosonar signals. We suggest that some combinationsensitive response properties in the IC are well suited for analyses of social vocalizations.

\section{Spectral integration in ascending auditory pathways}

The spectral integration documented here differs from other forms of integration described previously in the auditory brainstem and midbrain. For instance, in comparison with auditory nerve fibers, some cochlear nucleus neurons display narrower frequency tuning, whereas others show broader frequency tuning, noise sensitivity, or facilitatory-inhibitory frequency interactions (Rhode and Greenberg, 1992a,b; Young et al., 1992; Jiang et al., 1996). In the IC, most neurons have frequency tuning curves that differ significantly in shape from those of auditory nerve fibers (Ehret and Moffat, 1985a; Ehret and Merzenich, 1988; Ramachandran et al., 1999). Among 


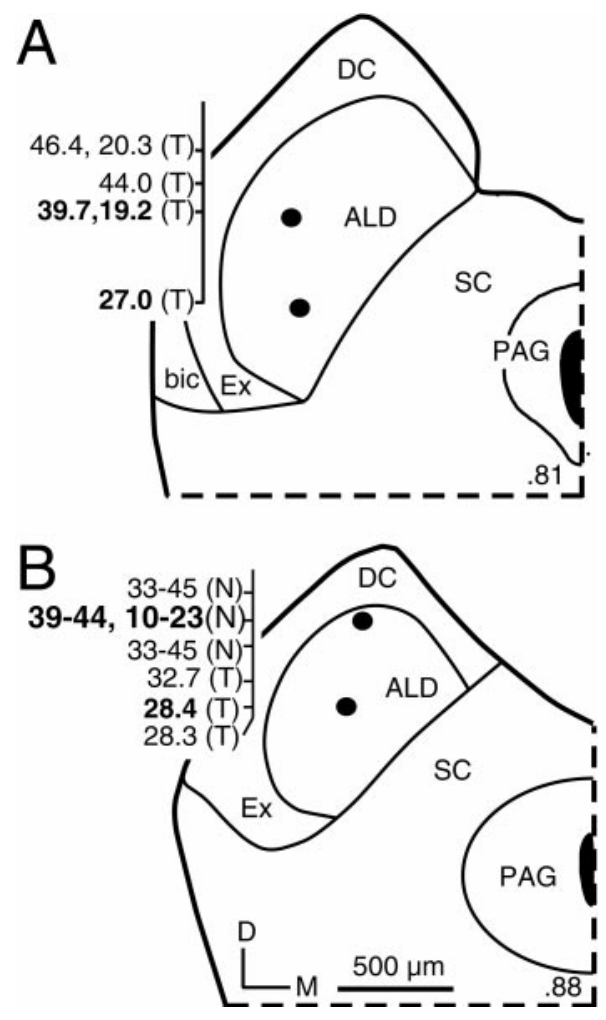

Figure 8. Locations of tone-responsive and noise-responsive recording sites in the $A L D$ of the ICC. All deposits of tracer shown here were made at single-unit recording sites indicated by filled circles in this figure and had frequency responses indicated in bold numbers to the left of the section. Most other responses illustrated in this figure were multiunit responses. For combination-sensitive responses, both the higher and lower best frequencies are reported. The number in the bottom right corner of each section indicates the location of the section along the caudal-to-rostral dimension of the IC. $A$, In the more caudal section, neurons tuned to the $32-47 \mathrm{kHz}$ band were tone responsive $(T)$. $B$, In the more rostral section, neurons tuned to this frequency band were noise responsive $(N)$ and combination sensitive. $P A G$, Periaqueductal gray.

IC neurons, local application of antagonists to inhibitory neurotransmitters alters frequency tuning and selectivity for frequency sweeps (Vater et al., 1992; Yang et al., 1992; Fuzessery and Hall, 1996), suggesting that mechanisms of spectral integration act in the IC as well as the cochlear nucleus. These studies generally demonstrate frequency integration that involves either integration of excitatory and/or inhibitory inputs tuned to relatively closely related frequencies or broad integration across a continuous band of frequencies that supplies inhibition on either side of an excitatory tuning curve. One exception is the multipeaked tuning curve, which may reflect dual excitatory inputs. However, such tuning curves are quite rare in the IC (Ehret and Moffat, 1985a; Casseday and Covey, 1992).

In contrast, the integration documented here is specific for widely separated frequency bands, usually an octave or more, suggesting input from two neural populations tuned to widely separated frequencies. These combinatorial properties, characterized by excitation, facilitation, or inhibition activated by distinct frequency bands of sounds, have typically been described in areas of the auditory forebrain (cortex or thalamus) outside of tonotopically organized regions (Suga et al., 1978, 1983; Fuzessery and Feng, 1983; Margoliash and Fortune, 1992; Olsen, 1992; Rauschecker et al., 1995; Ohlemiller et al., 1996; Doupe, 1997). This has supported the view that higher-order processing of complex sounds performed by combinatorial neurons is characteristic of forebrain auditory centers, particularly nontonotopic regions (Olsen, 1992; Winer et al., 1995; Rauschecker, 1998).

Although spectral combinatorial responses may be more common or more highly organized in nontonotopic auditory cortical areas, they also occur in tonotopically organized auditory cortex. In mustached bats, combination-sensitive responses are common in primary auditory cortex (Fitzpatrick et al., 1993; Kanwal et al., 1999). Increasingly, similar spectral combinatorial properties involving facilitation or inhibition have been described in primary auditory cortices of cats and primates. These properties include multipeaked excitatory tuning curves (Sutter and Schreiner, 1991), multiple inhibitory tuning curves (Sutter et al., 1999), and timedependant facilitatory and inhibitory interactions between different spectral elements (Brosch et al., 1999; Kadia et al., 2000). Viewed across species, the data suggest that many neurons in the primary auditory cortex use facilitatory and/or inhibitory interactions between distinct spectral sensitivities to analyze complex acoustic signals.

Do spectral combinatorial properties originate in the primary auditory cortex? In most species, observations of cortical combinatorial responses are not matched by similar observations in the auditory midbrain or thalamus. However, these latter regions have not been systematically examined using unanesthetized or lightly anesthetized preparations, test paradigms, or natural stimuli that may be related to recent observations of combinatorial properties in the primary auditory cortex. Application of these methods may reveal additional spectral combinatorial responses. For example, previous studies of cat and monkey MGB found that the complexity of frequency tuning curves and frequency organization increased when obtained from unanesthetized or lightly anesthetized animals (Allon et al., 1981; Morel et al., 1987). Recent studies of the ventral division of the MGB support the presence of complex inhibitory frequency tuning (Imig et al., 1997).

In the mustached bat, previous studies and the current results show that combinatorial response properties are created within the tonotopically organized ascending pathway below the auditory forebrain. In IC representations of frequencies within higher harmonics of the bat's biosonar signal, $\sim 75 \%$ of neurons display combination-sensitive response properties (Portfors and Wenstrup, 1999a). There are many similarities in combination-sensitive responses between those populations and the neurons described here. All are characterized by neural interactions activated by sound in two distinct frequency bands. These interactions are sensitive to the relative timing of two signals in the range of milliseconds or tens of milliseconds. Finally, each of the IC populations of combination-sensitive neurons displays facilitatory and inhibitory interactions between two frequency inputs, with a range in the strength of interactions.

Most combination-sensitive responses in the mustached bat appear to originate in one nucleus, the ICC. Thus, patterns of inputs to combination-sensitive IC neurons from the cochlear and lateral lemniscal nuclei, the lack of combination-sensitive responses in the lateral lemniscal nuclei, and the ability of strychnine to eliminate combination-sensitive facilitation in IC neurons all favor the hypothesis that most sonar-related combination-sensitive properties are constructed in the ICC (Leroy and Wenstrup, 1999; Portfors and Wenstrup, 1999b; Wenstrup et al., 1999, 2000). On the basis of the similarities among combination-sensitive response properties, we hypothesize that nonsonar neurons also originate in the ICC and depend on similar neuronal mechanisms.

Despite physiological and possible mechanistic similarities with sonar-related combination-sensitive neurons, we believe the present results have broader significance. Although sonar-related combination-sensitive neurons may function in other behavioral contexts such as social communication (Ohlemiller et al., 1996; Esser et al., 1997), their functional roles are closely linked to analyses of information about sonar objects, e.g., distance and movement (O’Neill and Suga, 1982; Suga et al., 1983; Olsen and Suga, 1991a,b). Their presence in the IC might be regarded as a specialized neural feature unique to a highly specialized behavior. The combination-sensitive response properties described here do not function in biosonar behavior. We conclude that the presence of combination-sensitive properties in the IC is not a sonar-related 
specialization. Instead, such response properties may be a general feature of analyses of complex sounds in the IC.

\section{Possible roles of combination-sensitive response properties}

The nonsonar neurons described here do not analyze sonar echoes but may instead analyze other complex acoustic signals of interest to mustached bats. These signals include social vocalizations of mustached bats (Kanwal et al., 1994), sonar and social vocalizations of other bat species (Goodwin, 1970), clicks produced by moths to deter bat predation (Dunning and Roeder, 1965; Goldman and Henson, 1977), and other animal-generated sounds. The complex spectral responses of nonsonar neurons could participate in both identification and localization of these signals. However, this discussion focuses on their potential role in discriminating among the mustached bat's social vocalizations because there is a striking correspondence between the dual frequency sensitivities of the neurons and spectral peaks occurring in several mustached bat social vocalizations [Kanwal et al. (1994), their Fig. 13].

We predict that spectral and temporal response properties of nonsonar combination-sensitive neurons should provide selectivity among the mustached bat's repertoire of social vocalizations. In this regard, the most obvious response property is dual frequency selectivity, which will restrict strong responses to those calls with significant energy in the $10-23$ and $32-47 \mathrm{kHz}$ bands. Additional selectivity could result from the harmonic relationship between lower and higher best frequencies of nonsonar neurons. For example, in some neurons the two best frequencies are tuned near a fundamental-second harmonic (1:2) relationship, so these neurons would respond well to constant frequency $(\mathrm{CF})$ signals with their fundamental at the neuron's lower best frequency. Other neurons, not tuned in a 1:2 harmonic relationship, should respond better to social vocalizations that have low-frequency fundamentals with many harmonics [e.g., short, quasi CF signals (Kanwal et al., 1994)] or have broad bands. Finally, a major source of variation among social vocalizations of the same category is a call's fundamental frequency (Kanwal et al., 1994). A population of such neurons, tuned to different frequency combinations, could distinguish among different versions of the same call, possibly emitted by different individuals.

A distinctive feature of many nonsonar combination-sensitive neurons is their requirement for bandpass noise in each of the $10-23$ and $32-47 \mathrm{kHz}$ bands. These neurons may respond preferentially to a class of mustached bat vocalizations containing noise bursts (Kanwal et al., 1994). One such call, a rectangular broadband noise burst used in agonistic interactions among individual mustached bats (Gupta et al., 1998), should effectively stimulate noisesensitive facilitated neurons. These communication signals may elicit fixed behavioral responses and thus on theoretical grounds may be most appropriate for processing within the IC (Casseday and Covey, 1996).

The above considerations suggest that nonsonar neurons may show selectivity among social vocalizations, something that has been examined rarely in the IC (Ehret and Moffat, 1985b; Aitkin et al., 1994). Direct studies of responses of nonsonar, combinationsensitive neurons to the mustached bat's repertoire of social vocalizations will be required to test this prediction.

\section{REFERENCES}

Aitkin LM, Tran L, Syka J (1994) The responses of neurons in subdivisions of the inferior colliculus of cats to tonal, noise and vocal stimuli. Exp Brain Res 98:53-64.

Allon N, Yeshurun Y, Wollberg Z (1981) Responses of single cells in the medial geniculate body of awake squirrel monkeys. Exp Brain Res 41:222-232.

Brosch M, Schulz A, Scheich H (1999) Processing of sound sequences in macaque auditory cortex: response enhancement. J Neurophysiol $82: 1542-1559$.

Casseday JH, Covey E (1992) Frequency tuning properties of neurons in the inferior colliculus of an FM bat. J Comp Neurol 319:34-50.

Casseday JH, Covey E (1996) A neuroethological theory of the operation of the inferior colliculus. Brain Behav Evol 47:311-336.

Doupe AJ (1997) Song- and order-selective neurons in the songbird ante- rior forebrain and their emergence during vocal development. J Neurosci 17:1147-1167.

Dunning DC, Roeder KD (1965) Moth sounds and the insect-catching behavior of bats. Science 147:173-174.

Ehret G, Merzenich MM (1988) Complex sound analysis (frequency resolution, filtering and spectral integration) by single units of the inferior colliculus of the cat. Brain Res 472:139-163.

Ehret G, Moffat AJM (1985a) Inferior colliculus of the house mouse. II. Single unit responses to tones, noise and tone-noise combinations as a function of sound intensity. J Comp Physiol [A] 156:619-635.

Ehret G, Moffat AJM (1985b) Inferior colliculus of the house mouse. III. Response probabilities and thresholds of single units to synthesized mouse calls compared to tone and noise bursts. J Comp Physiol [A] 156:637-644.

Esser KH, Condon CJ, Suga N, Kanwal JS (1997) Syntax processing by auditory cortical neurons in the FM-FM area of the mustached bat Pteronotus parnellii. Proc Natl Acad Sci USA 94:14019-14024.

Fitzpatrick DC, Kanwal JS, Butman JA, Suga N (1993) Combinationsensitive neurons in the primary auditory cortex of the mustached bat. J Neurosci 13:931-940.

Frisina RD, O'Neill WE, Zettel ML (1989) Functional organization of mustached bat inferior colliculus. II. Connections of the $\mathrm{FM}_{2}$ region. J Comp Neurol 284:85-107.

Fuzessery ZM, Feng AS (1983) Mating call selectivity in the thalamus and midbrain of the leopard frog (Rana p. pipiens): single and multiunit analyses. J Comp Physiol [A] 150:333-344.

Fuzessery ZM, Hall JC (1996) Role of GABA in shaping frequency tuning and creating FM sweep selectivity in the inferior colliculus. J Neurophysiol 76:1059-1073.

Goldman LJ, Henson Jr OW (1977) Prey recognition and selection by the constant frequency bat, Pteronotus p. parnellii. Behav Ecol Sociobiol 2:411-419.

Goodwin RE (1970) The ecology of Jamaican bats. J Mammal 51:571-579.

Gupta P, Dietz N, Kanwal JS (1998) Vocal communication and stereotypic behavior patterns in the mustached bat, Pteronotus parnellii. Assoc Res Otolaryngol Abstr 21:141.

Imig TJ, Poirier P, Irons WA, Samson FK (1997) Monaural spectral contrast mechanism for neural sensitivity to sound direction in the medial geniculate body of the cat. J Neurophysiol 78:2754-2771.

Jiang D, Palmer AR, Winter IM (1996) Frequency extent of two-tone facilitation in onset units in the ventral cochlear nucleus. J Neurophysiol 75:380-395

Kadia S, Snider R, Wang X (2000) Influence of stimulus components placed outside classical receptive field reveals harmonic structure of the auditory system. Assoc Res Otolaryngol Abstr 23:54.

Kanwal JS, Matsumura S, Ohlemiller K, Suga N (1994) Analysis of acoustic elements and syntax in communication sounds emitted by mustached bats. J Acoust Soc Am 96:1229-1254.

Kanwal JS, Fitzpatrick DC, Suga N (1999) Facilitatory and inhibitory frequency tuning of combination-sensitive neurons in the primary auditory cortex of mustached bats. J Neurophysiol 82:2327-2345.

Keating AW, Henson Jr OW, Henson MM, Lancaster WC, Xie DH (1994) Doppler-shift compensation by the mustached bat: quantitative data. J Exp Biol 188:115-129.

Kobler JB, Wilson BS, Henson Jr OW, Bishop AL (1985) Echo intensity compensation by echolocating bats. Hear Res 20:99-108.

Lancaster WC, Keating AW, Henson Jr OW (1992) Ultrasonic vocalizations of flying bats monitored by radiotelemetry. J Exp Biol 173:43-58.

Leroy SA, Wenstrup JJ (1999) Role of inhibitory neurotransmitters in responses of combination-sensitive neurons of the mustached bat's inferior colliculus. Assoc Res Otolaryngol Abstr 22:219.

Margoliash D, Fortune ES (1992) Temporal and harmonic combinationsensitive neurons in the zebra finch's HVc. J Neurosci 12:4309-4326.

Mittmann DH, Wenstrup JJ (1995) Combination-sensitive neurons in the inferior colliculus. Hear Res 90:185-191.

Morel A, Rouiller E, de Ribaupierre Y, de Ribaupierre F (1987) Tonotopic organization in the medial geniculate body (MGB) of lightly anesthetized cats. Exp Brain Res 69:24-42.

Norberg UM (1987) Wing form and flight mode in bats. In: Recent advances in the study of bats (Fenton MB, Racey P, Rayner JMV, eds), pp 43-56. Cambridge, UK: Cambridge UP.

Ohlemiller KK, Kanwal JS, Suga N (1996) Facilitative responses to species-specific calls in cortical FM-FM neurons of the mustached bat. NeuroReport 7:1749-1755.

Olsen JF (1992) High-order auditory filters. Curr Opin Neurobiol 2:489-497.

Olsen JF, Suga N (1991a) Combination-sensitive neurons in the medial geniculate body of the mustached bat: encoding of relative velocity information. J Neurophysiol 65:1254-1274.

Olsen JF, Suga N (1991b) Combination-sensitive neurons in the medial geniculate body of the mustached bat: encoding of target range information. J Neurophysiol 65:1275-1296.

O'Neill WE, Suga N (1979) Target range-sensitive neurons in the auditory cortex of the mustache bat. Science 203:69-73.

O’Neill WE, Suga N (1982) Encoding of target range and its representation in the auditory cortex of the mustached bat. J Neurosci 2:17-31.

O’Neill WE, Frisina RD, Gooler DM (1989) Functional organization of 
mustached bat inferior colliculus. I. Representation of FM frequency bands important for target ranging revealed by ${ }^{14} \mathrm{C}$-2-deoxyglucose autoradiography and single unit mapping. J Comp Neurol 284:60-84.

Portfors CV, Wenstrup JJ (1999a) Delay-tuned neurons in the inferior colliculus of the mustached bat: implications for analyses of target distance. J Neurophysiol 82:1326-1338.

Portfors CV, Wenstrup JJ (1999b) Origin of combination-sensitive neurons in the mustached bat: evidence from the nuclei of the lateral lemniscus. Soc Neurosci Abstr 25:396.

Ramachandran R, Davis KA, May BJ (1999) Single-unit responses in the inferior colliculus of decerebrate cats. I. Classification based on frequency response maps. J Neurophysiol 82:152-163.

Rauschecker JP (1998) Cortical processing of complex sounds. Curr Opin Neurobiol 8:516-521.

Rauschecker JP, Tian B, Hauser M (1995) Processing of complex sounds in the macaque nonprimary auditory cortex. Science 268:111-114.

Rhode WS, Greenberg S (1992a) Physiology of the cochlear nuclei. In: The mammalian auditory pathway: neurophysiology (Popper AN, Fay RR, eds), pp 94-152. New York: Springer.

Rhode WS, Greenberg S (1992b) Lateral suppression and inhibition in the cochlear nucleus of the cat. J Neurophysiol 71:493-514.

Schnitzler H-U (1970) Echoortung bei der Fledermaus Chilonycteris rubiginosa. Z Vergl Physiolog 68:25-38.

Schuller G, Covey E, Casseday JH (1991a) Auditory pontine gray: connections and response properties in the horseshoe bat. Eur $\mathrm{J}$ Neurosci 3:648-662.

Schuller G, O'Neill WE, Radtke-Schuller S (1991b) Facilitation and delay sensitivity of auditory cortex neurons in CF-FM bats, Rhinolophus rouxi and Pteronotus p. parnellii. Eur J Neurosci 3:1165-1181.

Schweizer H (1981) The connections of the inferior colliculus and the organization of the brainstem auditory system in the greater horseshoe bat (Rhinolophus ferrumequinum). J Comp Neurol 201:25-49.

Suga N (1996) Basic acoustic patterns and neural mechanisms shared by humans and animals for auditory perception: a neuroethologists's view. In: Auditory basis of speech perception (Ainsworth A, Greenberg S, eds), pp 31-38. Staffordshire, UK: Keele University.

Suga N, O'Neill WE, Manabe T (1978) Cortical neurons sensitive to combinations of information-bearing elements of biosonar signals in the mustache bat. Science 200:778-781.

Suga N, O’Neill WE, Kujirai K, Manabe T (1983) Specificity of combination-sensitive neurons for processing of complex biosonar signals in auditory cortex of the mustached bat. J Neurophysiol 49:1573-1626.

Suga N, Niwa H, Taniguchi I, Margoliash D (1987) The personalized auditory cortex of the mustached bat: adaptation for echolocation. J Neurophysiol 58:643-654.
Sussman HM, Frutcher D, Hilbert J, Sirosh J (1998) Linear correlates in the speech signal: the orderly output constraint. Behav Brain Sci 21:241-299.

Sutter ML, Schreiner CE (1991) Physiology and topography of neurons with multipeaked tuning curves in cat primary auditory cortex. J Neurophysiol 65:1207-1226.

Sutter ML, Schreiner CE, McLean M, O'Connor KN, Loftus WC (1999) Organization of inhibitory frequency receptive fields in cat primary auditory cortex. J Neurophysiol 82:2358-2371.

Vater M, Habbicht H, Kossl M, Grothe B (1992) The functional role of GABA and glycine in monaural and binaural processing in the inferior colliculus of horseshoe bats. J Comp Physiol [A] 171:541-553.

Wenstrup JJ (1999) Frequency organization and responses to complex sounds in the medial geniculate body of the mustached bat. J Neurophysiol 82:2528-2544.

Wenstrup JJ, Grose CD (1995) Inputs to combination-sensitive neurons in the medial geniculate body of the mustached bat: the missing fundamental. J Neurosci 15:4693-4711.

Wenstrup JJ, Larue DT, Winer JA (1994) Projections of physiologically defined subdivisions of the inferior colliculus in the mustached bat: targets in the medial geniculate body and extrathalamic nuclei. J Comp Neurol 346:207-236.

Wenstrup JJ, Mittmann DH, Grose CD (1999) Inputs to combinationsensitive neurons in the inferior colliculus. J Comp Neurol 409:509-528.

Wenstrup JJ, Leroy SA, Portfors C, Grose CD (2000) Neural mechanisms underlying the analysis of target distance. In: Echolocation in bats and dolphins (Thomas J, Moss C, Vater M, eds). Chicago: University of Chicago, in press.

Winer JA, Larue DT, Pollak GD (1995) GABA and glycine in the central auditory system of the mustache bat: structural substrates for inhibitory neuronal organization. J Comp Neurol 355:317-353.

Yan J, Suga N (1996) The midbrain creates and the thalamus sharpens echo-delay tuning for the cortical representation of target-distance information in the mustached bat. Hear Res 93:102-110.

Yang L, Pollak GD, Resler C (1992) GABAergic circuits sharpen tuning curves and modify response properties in the mustache bat inferior colliculus. J Neurophysiol 68:1760-1774.

Young ED, Spirou GA, Rice JJ, Voigt HF (1992) Neural organization and responses to complex stimuli in the dorsal cochlear nucleus. Philos Trans R Soc Lond [Biol] 336:407-413.

Zook JM, Winer JA, Pollak GD, Bodenhamer RD (1985) Topology of the central nucleus of the mustache bat's inferior colliculus: correlation of single unit response properties and neuronal architecture. J Comp Neurol 231:530-546. 